Contemporary Mathematics

\title{
A survey on maximal green sequences
}

\author{
Bernhard Keller, with an appendix by Laurent Demonet
}

\begin{abstract}
Maximal green sequences appear in the study of Fomin-Zelevinsky's cluster algebras. They are useful for computing refined Donaldson-Thomas invariants, constructing twist automorphisms and proving the existence of theta bases and generic bases. We survey recent progress on their existence and properties and give a representation-theoretic proof of Greg Muller's theorem stating that full subquivers inherit maximal green sequences. In the appendix, Laurent Demonet describes maximal chains of torsion classes in terms of bricks generalizing a theorem by Igusa.
\end{abstract}

\section{Introduction}

A quiver is an oriented graph. Quiver mutation is an elementary operation on quivers. It is the basic combinatorial ingredient of Fomin-Zelevinsky's definition of cluster algebras [46]. In this definition, one recursively constructs generators for the cluster algebra by repeatedly mutating an initial seed $(Q, x)$ consisting of a quiver $Q$ and a set $x$ of indeterminates associated with the vertices of $Q$. The construction process is recorded in a graph, the exchange graph, whose vertices are the seeds obtained from $(Q, x)$ by iterated mutation and whose edges correspond to mutations. By definition, the edges of the exchange graph are unoriented. However, it was noticed early on [90] that there is a natural partial order on seeds whose minimal inclusions correspond to edges of the exchange graph, which thus becomes oriented. For example, for a linearly oriented quiver of type $A_{n}$, the poset of seeds is the $n$th Tamari lattice $[71,110]$.

Maximal green sequences were invented in [74] (and became part of [77]) but are already implicit in the work of Gaiotto-Moore-Neitzke [51] (published in [52]). A maximal green sequence is a (finite) path in the oriented exchange graph from the unique smallest element to the unique largest element. Not all quivers have maximal green sequences but they do exist for important classes of quivers and their existence has important consequences: it yields explicit formulas for Kontsevich-Soibelman's refined Donaldson-Thomas invariant associated [81] with the quiver, for the twist automorphism [58] of the associated cluster algebra and it is a sufficient condition for the existence of a theta basis [60] and a generic basis [103] in the upper cluster algebra.

In section 2, we review the purely combinatorial definitions of mutation and green mutation leading to the notion of (maximal) green sequence (and, more generally, reddening sequence). In section 3 , we describe the applications of maximal green sequences mentioned above. We then report on results concerning the existence and non existence of maximal green sequences (section 4). In particular, we state Greg Muller's theorem to the effect that full subquivers inherit maximal green sequences. In the final section 5, we give a proof of Muller's theorem based on recent results in the study of torsion classes [33]. In the appendix, Laurent Demonet establishes a bijection between maximal chains of torsion classes and maximal forward Hom-orthogonal sequences of bricks over a finite-dimensional algebra generalizing a theorem proved by Igusa [65] for representation-finite Jacobi algebras.

2010 Mathematics Subject Classification. 13F60 (primary); 14N35, 18E40, 18E30 (secondary).

Key words and phrases. Cluster algebra, maximal green sequence, Donaldson-Thomas invariant, theta basis, generic basis, twist automorphisms, torsion class. 


\section{Acknowledgments}

I thank Laurent Demonet for his explanations of the results of [33], his help with section 5 and for contributing the appendix. I am grateful to Daniel Labardini-Fragoso for helpful discussions and for reference [86]. An abridged version of this note was presented at the ICRA 18 in Prague in August 2018. I thank the organisers for a wonderful conference and for considering this material for the proceedings. I am indebted to Zheng Hua for pointing out a misquotation in section 6 of a previous version of this note and to Jiarui Fei for alerting me to reference [41]. I thank Volker Genz for pointing out an incorrect formulation of Remark 4.3 in the published version of this note.

\section{Mutation and green mutation}

2.1. Quiver mutation. A quiver is an oriented graph, i.e. a quadruple $Q=\left(Q_{0}, Q_{1}, s, t\right)$ formed by a set of vertices $Q_{0}$, a set of arrows $Q_{1}$ and two maps $s$ and $t$ from $Q_{1}$ to $Q_{0}$ which send an arrow $\alpha$ respectively to its source $s(\alpha)$ and its target $t(\alpha)$. In practice, a quiver is given by a picture as in the following example

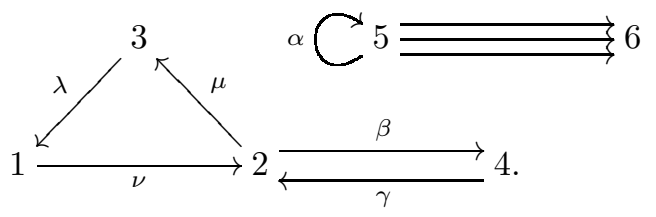

An arrow $\alpha$ whose source and target coincide is a loop; a 2-cycle is a pair of distinct arrows $\beta$ and $\gamma$ such that $s(\beta)=t(\gamma)$ and $t(\beta)=s(\gamma)$. Similarly, one defines $n$-cycles for any positive integer $n$. A vertex $i$ of a quiver is a source (respectively a sink) if there is no arrow with target $i$ (respectively with source $i$ ).

By convention, in the sequel, by a quiver, we always mean a finite quiver without loops nor 2 -cycles whose set of vertices is the set of integers from 1 to $n$ for some $n \geqslant 1$. Up to an isomorphism fixing the vertices, such a quiver $Q$ is given by the skew-symmetric matrix $B=B_{Q}$ whose coefficient $b_{i j}$ is the difference between the number of arrows from $i$ to $j$ and the number of arrows from $j$ to $i$ for all $1 \leqslant i, j \leqslant n$. Conversely, each skew-symmetric matrix $B$ with integer coefficients comes from a quiver. follows:

Let $Q$ be a quiver and $k$ a vertex of $Q$. The mutation $\mu_{k}(Q)$ is the quiver obtained from $Q$ as

1) for each subquiver $i \stackrel{\beta}{\longrightarrow} k \stackrel{\alpha}{\longrightarrow} j$, we add a new arrow $[\alpha \beta]: i \rightarrow j$;

2) we reverse all arrows with source or target $k$;

3 ) we remove the arrows in a maximal set of pairwise disjoint 2-cycles.

For example, if $k$ is a source or a sink of $Q$, then the mutation at $k$ simply reverses all the arrows incident with $k$. In general, if $B$ is the skew-symmetric matrix associated with $Q$ and $B^{\prime}$ the one associated with $\mu_{k}(Q)$, we have

$$
b_{i j}^{\prime}= \begin{cases}-b_{i j} & \text { if } i=k \text { or } j=k \\ b_{i j}+\operatorname{sgn}\left(b_{i k}\right) \max \left(0, b_{i k} b_{k j}\right) & \text { else. }\end{cases}
$$

This is the matrix mutation rule introduced by Fomin-Zelevinsky in [46], cf. also [48]. It applies more generally to skew-symmetrizable matrices, which correspond to valued quivers, cf. section 3.3 of $[78]$.

One checks easily that $\mu_{k}$ is an involution. For example, the quivers

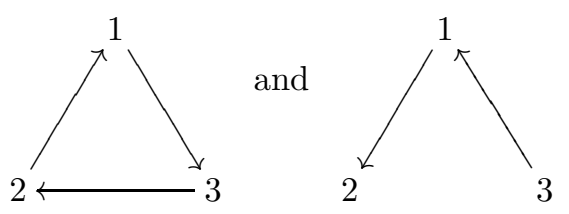




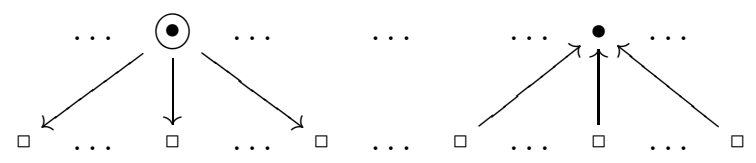

Figure 1. Green $\bullet$ and red $\bullet$ vertices in $R$

are linked by a mutation at the vertex 1 . Notice that these quivers are drastically different: The first one is a cycle, the second one the Hasse diagram of a linearly ordered set.

Two quivers are mutation equivalent if they are linked by a finite sequence of mutations. For example, it is an easy exercise to check that any two orientations of a tree are mutation equivalent. Using the quiver mutation applet [76] or the Sage package [95] one can check that the following three quivers are mutation equivalent
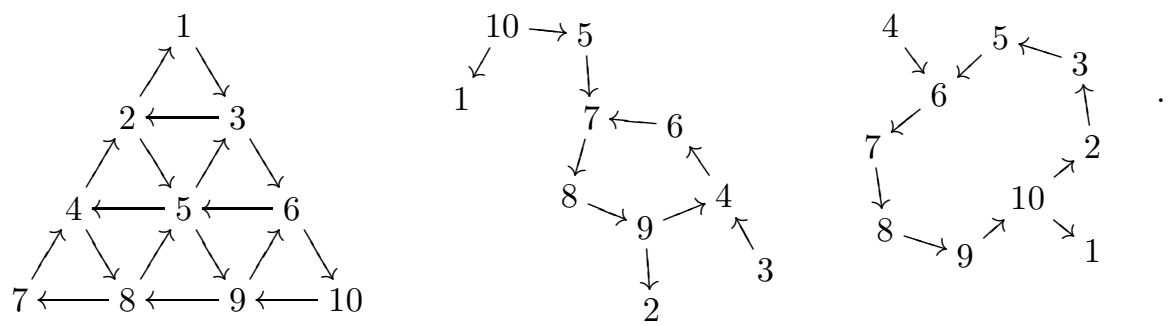

The common mutation class of these quivers contains 5739 quivers (up to isomorphism). The mutation class of 'most' quivers is infinite. The classification of the quivers having a finite mutation class was achieved by Felikson-Shapiro-Tumarkin [43, 42]: in addition to the quivers associated with triangulations of surfaces (with boundary and marked points, cf. [45]) the list contains 11 exceptional quivers, the largest of which is in the mutation class of the quivers (2.1.3).

2.2. Green quiver mutation. Let $Q$ be a quiver. The framed quiver $\widehat{Q}$ is obtained from $Q$ by adding, for each vertex $i$, a new vertex $i^{\prime}$ and a new arrow $i \rightarrow i^{\prime}$. For example, if $Q$ is the quiver $1 \rightarrow 2$, then the framed quiver $\widehat{Q}$ is

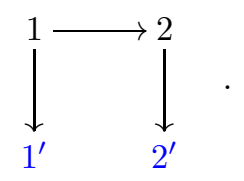

The new vertices $i^{\prime}$ are called frozen vertices, because we never mutate at them. Now suppose that we have transformed $\hat{Q}$ into a quiver $R$ by a finite sequence of mutations (at non frozen vertices). A non frozen vertex $i$ is green in $R$ if it is not the target of any arrows $j^{\prime} \rightarrow i$ from frozen vertices $j^{\prime}$ in $R$. It is red if it is not the source of any arrows $i \rightarrow j^{\prime}$ to frozen vertices of $R$, cf. Figure 1 .

Theorem 2.3 (Derksen-Weyman-Zelevinsky [36]). Each non frozen vertex of $R$ is either green or red.

The proof is based on the theory of mutations of quivers with potential and their decorated representations developed in [35, 36]. Alternative proofs of the theorem were given in [101] (via the cluster category) and in [97] (via Donaldson-Thomas theory). An important generalization to valued quivers is proved [60]. As shown in [98], the theorem is central in the theory of cluster algebras.

The $c$-vector $\alpha_{i} \in \mathbb{Z}^{n}$ associated with a non frozen vertex $i$ of $R$ is the integer vector whose $j$ th component is the difference between the number of arrows from $i$ to $j^{\prime}$ minus the number of arrows from $j^{\prime}$ to $i$ in $R$. By the theorem, each $c$-vector has either all components $\geqslant 0$ or all components $\leqslant 0$ (sign-coherence of $c$-vectors). The following definition was first given in [74, 77]. A sequence 


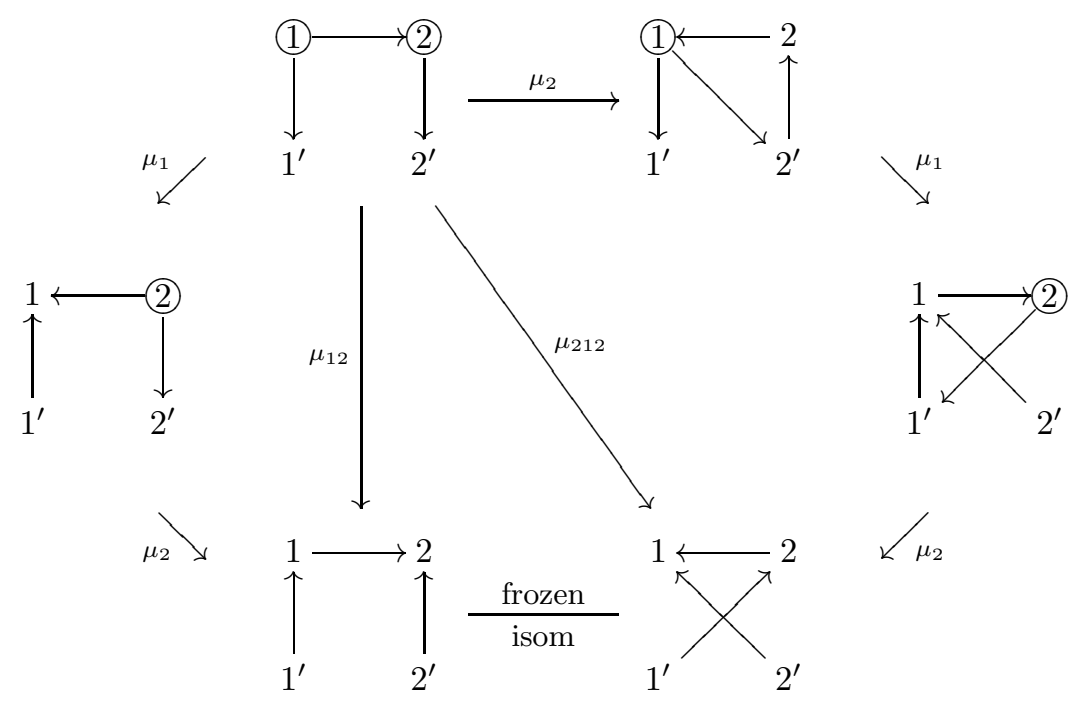

Figure 2. The two maximal green sequences for $\vec{A}_{2}$

$\mathbf{i}=\left(i_{1}, \ldots, i_{N}\right)$ of vertices is green if, for each $1 \leqslant t \leqslant N$, the vertex $i_{t}$ is green in the partially mutated quiver

$$
\mu_{i_{t-1}} \ldots \mu_{i_{2}} \mu_{i_{1}}(\widehat{Q}) .
$$

It is maximal green if moreover all non frozen vertices of $\mu_{\mathbf{i}}(\hat{Q})$ are red. It is reddening [75] (or green-to-red [93]) if it is not necessarily green but all non frozen vertices of $\mu_{\mathbf{i}}(\hat{Q})$ are red.

In Figure 2, we see that the quiver $\vec{A}_{2}$ has exactly two maximal green sequences: $(1,2)$ and $(2,1,2)$ (green vertices are encircled). The final quivers in the two sequences are isomorphic by a frozen isomorphism (i.e. an isomorphism which fixes the frozen vertices) to the coframed quiver $\check{Q}$, which is obtained from $Q$ by adding, for each vertex $i$, a new vertex $i^{\prime}$ and an arrow $i^{\prime} \rightarrow i$. This is a general phenomenon:

Proposition 2.4 (Prop. 2.10 of [11]). Suppose that $Q$ admits a reddening sequence $\mathbf{i}$. Then there is a unique isomorphism $\mu_{\mathbf{i}}(\widehat{Q}) \stackrel{\sim}{\rightarrow} \breve{Q}$ fixing the frozen vertices and sending a non frozen vertex $i$ to $\sigma(i)$ for a unique permutation $\sigma$ of the vertices of $Q$.

For maximal green sequences of quivers in the mutation class of $A_{n}$, the permutation $\sigma$ is studied in $[56,69]$. In general, it remains mysterious.

It is a fact that large classes of quivers appearing in Lie theory and higher Teichmüller theory do admit maximal green sequences. We refer to section 4 below for classes of examples. This is interesting because of the applications sketched in the next section.

\section{Applications}

3.1. Refined Donaldson-Thomas invariants. The quantum dilogarithm series is defined by

$$
\begin{aligned}
\mathbb{E}(y) & =1+\frac{q^{1 / 2}}{q-1} \cdot y+\cdots+\frac{q^{n^{2} / 2} y^{n}}{\left(q^{n}-1\right)\left(q^{n}-q\right) \cdots\left(q^{n}-q^{n-1}\right)}+\cdots \\
& \in \mathbb{Q}\left(q^{1 / 2}\right)[[y]],
\end{aligned}
$$

where $q^{1 / 2}$ is an indeterminate whose square is denoted by $q$ and $y$ is an indeterminate. This series is a classical object with many remarkable properties, cf. for example [117]. 
Let $Q$ be a quiver with $n$ vertices. Let $\lambda_{Q}: \mathbb{Z}^{n} \times \mathbb{Z}^{n} \rightarrow \mathbb{Z}$ be the bilinear antisymmetric form associated with the matrix $B_{Q}$. Define the complete quantum affine space as the algebra

$$
\widehat{\mathbb{A}}_{Q}=\mathbb{Q}\left(q^{1 / 2}\right)\left\langle\left\langle y^{\alpha}, \alpha \in \mathbb{N}^{n} \mid y^{\alpha} y^{\beta}=q^{1 / 2 \lambda(\alpha, \beta)} y^{\alpha+\beta}\right\rangle\right\rangle .
$$

This is a slightly non commutative deformation of an ordinary commutative power series algebra in the $n$ indeterminates $y_{i}=y^{e_{i}}$, where $e_{i}$ is the $i$ th vector of the standard basis of $\mathbb{Z}^{n}$. We write $\mathbb{A}_{Q}$ for the non completed variant of $\widehat{\mathbb{A}}_{Q}$. For a sequence $\mathbf{i}=\left(i_{1}, \ldots, i_{N}\right)$ of vertices of $Q$, we define

$$
\mathbb{E}_{Q, \mathbf{i}}=\mathbb{E}\left(y^{\varepsilon_{1} \beta_{1}}\right)^{\varepsilon_{1}} \cdots \mathbb{E}\left(y^{\varepsilon_{N} \beta_{N}}\right)^{\varepsilon_{N}},
$$

where the product is taken in $\widehat{\mathbb{A}}_{Q}$, the vector $\beta_{t}$ is the $c$-vector associated with the vertex $i_{t}$ of the partially mutated quiver

$$
\mu_{i_{t-1}} \ldots \mu_{i_{1}}(\widehat{Q})
$$

and $\varepsilon_{i}$ is the common sign of its entries, $1 \leqslant t \leqslant N$. Notice that by the sign-coherence, each factor does belong to $\widehat{\mathbb{A}}_{Q}$.

Let $R D T_{Q} \in \widehat{\mathbb{A}}_{Q}$ denote the refined Donaldson-Thomas invariant of $Q$ constructed by Kontsevich-Soibelman $[81,83,84]$. Notice that their construction has not yet been made completely rigorous due to technical difficulties arising from the fact that the potentials needed are infinite linear combinations of cycles. The following theorem was the motivation for [74]. Independently, it was discovered in the study of the BPS spectrum by Gaiotto-Moore-Neitzke in [51] and used in the physics literature, for example in $[2,25]$, cf. $[116]$ and the references given there.

THEOREM 3.2. If $Q$ admits a reddening sequence $\mathbf{i}$, we have

$$
R D T_{Q}=\mathbb{E}_{Q, \mathbf{i}} \text { in } \widehat{\mathbb{A}}_{Q} .
$$

The theorem is proved in section 7 of [73] under the assumption that $R D T_{Q}$ is well-defined. It implies in particular that the right hand side is independent of the choice of $\mathbf{i}$. This was conjectured in [96] and can be proved rigorously using the theory of cluster algebras and their additive categorification, cf. section 7.11 of $[73]$.

Thanks to the theorem, each pair $\left(\mathbf{i}, \mathbf{i}^{\prime}\right)$ of reddening sequences yields a quantum dilogarithm identity. For example, the two maximal green sequences of the quiver $Q=\vec{A}_{2}$ yield the pentagon identity

$$
\mathbb{E}\left(y_{1}\right) \mathbb{E}\left(y_{2}\right)=\mathbb{E}\left(y_{2}\right) \mathbb{E}\left(q^{-1 / 2} y_{1} y_{2}\right) \mathbb{E}\left(y_{1}\right)
$$

due to [39] and [40], cf. [112] for a recent account. Analogous pairs of maximal green sequences exist for all Dynkin quivers and yield generalizations of the pentagon identity due to Reineke [105], cf. [77]. These identities further generalize to square products [79] of Dynkin quivers, cf. [77]. For pairs $\left(A_{n}, A_{m}\right)$, an alternative geometric proof of the identities is given by Allman-Rimanyi [3]. We refer to section 5 of [75] for more examples.

One may ask whether all the quantum dilogarithm identities obtained from the theorem are in fact consequences of the pentagon identity. This is not the case. Counterexamples based on [45] can be found in [80]. However, it does hold if $Q$ is an affine ayclic quiver, as shown by Hermes-Igusa [62].

In [59], Goncharov-Shen construct maximal green sequences for a large class of quivers appearing in higher Teichmüller theory and apply the theorem to obtain the corresponding DonaldsonThomas invariants. Similar results for Grassmannians and double Bruhat cells are due to Weng $[115,113,114]$ and for double Bott-Samelson cells to Shen-Weng [109].

3.3. Twist automorphisms. Let $Q$ be a quiver admitting a reddening sequence i. Let $\sigma$ be the permutation of Prop. 2.4. Then the composition of the mutation sequence $\mu_{\mathbf{i}}$ with the permutation $\sigma$ transforms the initial seed $(Q, x)$ of the cluster algebra $\mathcal{A}_{Q}$ into a seed of the form $(Q, u)$ and thus yields an automorphism

$$
\mathrm{tw}: \mathcal{A}_{Q} \stackrel{\sim}{\rightarrow} \mathcal{A}_{Q}, x_{i} \mapsto u_{i}
$$

called the twist automorphism of $\mathcal{A}_{Q}$. 
BERNHARD KELLER

From the independence of $\mathbb{E}_{Q, \mathbf{i}}$ of the choice of the reddening sequence $\mathbf{i}$, one can deduce that the twist automorphism tw is also independent of $\mathbf{i}$ ( $c f$. section 4 of [77] and section 6.4 of [78]).

As shown by Geiss-Leclerc-Schröer [58], for unipotent cells of Kac-Moody groups, the twist automorphism identifies with the chamber ansatz of Berenstein-Fomin-Zelevinsky [8]. It has found important applications in the work of Marsh-Scott [89], Muller-Speyer [94], Rietsch-Williams [106], Cautis-Williams [24], ...

3.4. The Fock-Goncharov conjectures. Let $Q$ be a valued quiver. The Langlands dual valued quiver $Q^{L}$ is obtained by reversing all the valuations of $Q$ (without changing the arrows). The skew-symmetrizable matrix $B_{Q^{L}}$ is the opposite transpose $-B_{Q}^{T}$ of $B_{Q}$. Thus, we have $Q=Q^{L}$ if $Q$ is an ordinary (i.e. equivalued) quiver. Suppose that $Q$ is obtained from an ice quiver $\widetilde{Q}$ by removing all the frozen vertices and all the arrows incident with them. Assume that the exchange matrix associated with $\widetilde{Q}$ is of maximal rank.

THEOREM 3.5 (Gross-Hacking-Keel-Kontsevich [60]). If $Q$ has a reddening sequence, then the Fock-Goncharov duality conjectures [44] hold for $Q$ and in particular the upper cluster algebra $\mathcal{U}_{\widetilde{Q}}$ admits a basis parametrized by the tropical points of the cluster Poisson variety associated with $\widetilde{Q}^{L}$.

The basis constructed by Gross-Hacking-Keel-Kontsevich is known as the theta basis. Generic bases were first considered by Dupont [38] for acyclic quivers. They are constructed using generic values of cluster characters [22, 100]. In [58], Geiss-Leclerc-Schröer showed that they exist for large classes of cluster algebras arising in Lie theory and coincide with Lusztig's dual semi-canonical bases. Plamondon showed in [102] that generic bases are also canonically parametrized by the tropical points of the cluster Poisson variety.

THEOREM 3.6 (Qin [103]). Let $Q$ be an (equivalued) quiver. If $Q$ has a reddening sequence, then the upper cluster algebra $\mathcal{U}_{\widetilde{Q}}$ admits a generic basis parametrized by the tropical points of the cluster Poisson variety associated with $\widetilde{Q}$.

Notice that these results concern the upper cluster algebra. It is expected that the existence of a maximal green, or reddening, sequence should have implications for the relationship between the cluster algebra and the upper cluster algebra. Notice however that this relationship depends on the choice of coefficients [41, Example 8.3] [20], whereas the oriented exchange graph, and thus the existence of a maximal green sequence, does not [26]. A conjecture on the precise relationship is formulated in [91].

\section{Existence and properties}

4.1. Existence. Suppose that $Q$ is an acyclic quiver. A source sequence for $Q$ is an enumeration of the vertices of $Q$ which is increasing for the partial order defined by the existence of a path. It is easy to check that each source sequence is a maximal green sequence for $Q$, cf. [11].

Recall that a valued quiver is cluster-finite (i.e. the associated cluster algebra has only finitely many cluster variables) iff it is mutation-equivalent to an orientation of a Dynkin diagram [47]. As observed in in [11], it is immediate from [27] that cluster-finite valued quivers have maximal green sequences.

For valued quivers mutation-equivalent to orientations of extended Dynkin diagrams, there are only finitely many maximal green sequences [12]. The same holds for (equally valued) acyclic quivers [70].

Two canonical maximal green sequences exist for the square product of two alternating valued Dynkin quivers, cf. section 5 of [75].

If $R$ is an ayclic quiver and $\widetilde{w}$ a reduced expression for an element of the Coxeter group associated with the underlying graph of $R$, there is a canonical quiver $Q$ associated with the pair $(R, \widetilde{w})$. It serves to obtain a cluster structure on the coordinate algebra of the unipotent cell associated with $w$ in the Kac-Moody group determined by $R$, cf. [5] and [57]. It is closely related to the (upper) cluster structures on Bruhat cells obtained in [9]. In section 13 of [57], GeissLeclerc-Schröer exhibit a canonical reddening sequence for $Q$ (we conjecture that it is actually maximal green). 
In analogy with the definition of a full subcategory, one defines a full subquiver $Q^{\prime}$ of a quiver $Q$ to be a subquiver containing all the arrows in $Q$ between any two of its vertices.

TheOREM 4.2 (Muller [93]). If $Q$ has a maximal green (resp. reddening) sequence, then each full subquiver $Q^{\prime} \subseteq Q$ has a maximal green (resp. reddening) sequence.

REMARK 4.3. Muller's proof shows that more precisely, starting from a maximal green sequence $\left(k_{1}, \ldots, k_{N}\right)$ for $Q$, one obtains a maximal green sequence for $Q^{\prime}$ as follows: Let $\left(c_{1}, \ldots, c_{N}\right)$ be the sequence of c-vectors associated with the given sequence $\left(k_{1}, \ldots, k_{N}\right)$. Form the subsequence $\left(c_{1}^{\prime}, \ldots, c_{M}^{\prime}\right)$ of $\left(c_{1}, \ldots, c_{N}\right)$ formed by those vectors supported on $Q^{\prime}$. Then there is a unique sequence of mutations $\left(k_{1}^{\prime}, \ldots, k_{M}^{\prime}\right)$ of $Q^{\prime}$ whose associated sequence of $c$-vectors is $\left(c_{1}^{\prime}, \ldots, c_{M}^{\prime}\right)$. The sequence $\left(k_{1}^{\prime}, \ldots, k_{M}^{\prime}\right)$ is the required maximal green sequence for $Q^{\prime}$.

Muller's proof uses the existence and uniqueness of the scattering diagram [82, 61] associated with a quiver [60]. We will give a representation-theoretic proof in section 5.

Let $Q$ be a quiver and $Q^{\prime}, Q^{\prime \prime}$ full subquivers. We say that $Q$ is a triangular extension of $Q^{\prime}$ by $Q^{\prime \prime}$ if the set of vertices of $Q$ is the disjoint union of the sets of vertices of $Q^{\prime}$ and $Q^{\prime \prime}$ and there are no arrows from vertices of $Q^{\prime \prime}$ to vertices of $Q^{\prime}$. After pioneering work in [56], the following theorem was proved in [23] using Muller's theorem 4.2.

THEOREM 4.4. If $Q$ is a triangular extension of $Q^{\prime}$ by $Q^{\prime \prime}$, then $Q$ has a maximal green sequence if and only if $Q^{\prime}$ and $Q^{\prime \prime}$ have maximal green sequences.

We refer to $[18,19]$ for recent extensions of this theorem. The case of mutation-finite quivers is treated in section 4.9 below.

4.5. Preservation under mutations. We have the following 'rotation lemma'.

Lemma 4.6 (Brüstle-Hermes-Igusa-Todorov [12]). If $\mathbf{i}=\left(i_{1}, \ldots, i_{N}\right)$ is maximal green (resp. reddening) for a quiver $Q$, then $\left(i_{2}, \ldots, i_{N}, k\right)$ is maximal green (resp. reddening) for $\mu_{i_{1}}(Q)$, where $k$ is the target of the unique arrow with source $i_{1}^{\prime}$ in $\mu_{\mathbf{i}}(\hat{Q})$.

ThEOREM 4.7 (Muller [93]). If $Q$ admits a reddening sequence, then each quiver mutationequivalent to $Q$ admits a reddening sequence.

However, the analogous statement for maximal green sequences is false, as we will see below.

4.8. Non existence. For three non negative integers $a, b, c$, denote by $Q_{a, b, c}$ the quiver with three vertices 1, 2, 3 and $a$ arrows from 1 to $2, b$ arrows from 2 to 3 and $c$ arrows from 3 to 1 (cf. [108] for a study of the case of valued quivers with 3 vertices). As shown in [11], the quiver $Q_{2,2,2}$ does not admit a maximal green sequence (nor does it admit a reddening sequence). Muller shows [93] that none of the quivers $Q_{a, b, c}$ with all three numbers $a, b, c \geqslant 2$ admits a maximal green sequence On the other hand, the quiver $Q_{2,2,3}$ is mutation-acyclic (the quiver $Q_{a, b, c}$ is mutationacyclic iff $\min (a, b, c)<2$ or $a^{2}+b^{2}+c^{2}-a b c>4$ as shown in [7]). Thus, the existence of a maximal green sequence is not preserved under mutation [93].

4.9. Existence and non existence for mutation-finite quivers. Generalizing the example of $Q_{2,2,2}$, Ladkani has shown in [87] that the quivers associated [45] with once-punctured surfaces of arbitrary genus (without boundary) do not admit reddening sequences (his proof is based on the work of Labardini-Fragoso [86] and Corollary 5.2 below). Seven shows in [107] that the quivers in the mutation class of the quiver $X_{7}$ (discovered in [34]) do not admit maximal green sequences (and presumably no reddening sequences either).

Partial results in the direction of the following theorem were obtained in $[2,17,21,29,56]$.

THEOREM 4.10 (Mills [92]). If $Q$ is a mutation-finite quiver, it has a maximal green sequence except if it comes from a once-punctured closed surface of genus $\geqslant 1$ or is in the mutation class of $X_{7}$.

Information on the length of the minimal length maximal green sequences for the quivers associated with annuli or punctured disks can be found in $[72,54,55]$. Maximal green sequences for minimal mutation-infinite quivers are studied in [88]. 


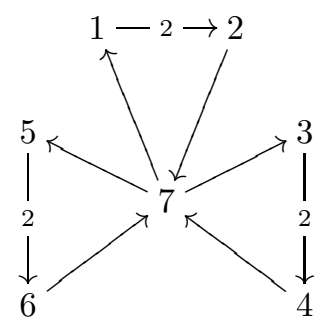

FiguRE 3 . The quiver $X_{7}$ (double arrows are marked with 2)

\section{Muller's theorem via representation theory}

Let $Q$ be a quiver admitting a maximal green sequence and $Q^{\prime} \subseteq Q$ a full subquiver. We wish to show that $Q^{\prime}$ has a maximal green sequence as well.

We recall the setup of Derksen-Weyman-Zelevinsky's theory of quivers with potentials and their mutations [35]. Let $k$ be an uncountable field. Let $k Q$ be the path algebra and $J$ the twosided ideal of $k Q$ generated by the arrows. Let $\widehat{k Q}$ be the completed path algebra, i.e. inverse limit of the finite-dimensional quotients $k Q / J^{n}, n \geqslant 1$. Let $W$ be a non degenerate potential, i.e. an element of $\widehat{k Q}$ which is an infinite linear combination of cycles of length $\geqslant 3$ such that the pair $(Q, W)$ can be mutated indefinitely without creating 2-cycles in the quiver component $Q^{\prime}$ of the mutated quiver with potential $\left(Q^{\prime}, W^{\prime}\right)$. Let $A$ be the Jacobi algebra of $(Q, W)$, i.e. the quotient of $\widehat{k Q}$ by the closed ideal generated by the cyclic derivatives $\partial_{\alpha} W, \alpha \in Q_{1}$. Notice that in general, the algebra $A$ is infinite-dimensional. Let $\bmod A$ be the category of finite-dimensional (right) $A$-modules. Recall that all finite-dimensional $A$-modules are nilpotent, i.e. annihilated by a sufficiently high power of the ideal $J$ (cf. section 10 of [35]). Therefore, the simple objects of $\bmod A$ are the simple modules $S_{i}$ associated with the vertices $i \in Q_{0}$. Thus, the abelian category $\bmod A$ is an Ext-finite length category with finitely many simple objects, cf. [50, 85].

Let $\mathcal{A}$ be an abelian category. For a class of objects $X$, we denote by $X^{\perp}$ the right orthogonal of $X$, i.e. the full subcategory of objects $Y$ such that $\operatorname{Hom}(X, Y)=0$ for all $X \in X$. We denote by $\operatorname{add}(X)$ the full subcategory formed by all direct summands of finite direct sums of objects of $X$. Recall that a torsion pair [37] in $\mathcal{A}$ is a pair of full subcategories $(\mathcal{T}, \mathcal{F})$ such that $\operatorname{Hom}(T, F)=0$ for all $T \in \mathcal{T}$ and $F \in \mathcal{F}$ and for each object $M$ of $\mathcal{A}$, there is a short exact sequence

$$
0 \longrightarrow T \longrightarrow M \longrightarrow F \longrightarrow 0
$$

with $T \in \mathcal{T}$ and $F \in \mathcal{F}$. In this case, $\mathcal{T}$ is called a torsion class. The torsion classes of the category of finite-dimensional modules over a finite-dimensional algebra are precisely the full subcategories closed under extensions and quotients [37]. A brick is an object whose endomorphism algebra is a division algebra.

The link between torsion classes and clusters is already implicit in Marsh-Reineke-Zelevinsky's [90]. It is made completely explicit by Ingalls-Thomas in [71] (in the acyclic case). As explained in section 7.6 of [78], the following theorem results from Nagao's work in [97]. For the case of acyclic quivers, cf. [104].

Theorem 5.1 (Nagao). Each green sequence $\mathbf{i}=\left(i_{1}, \ldots, i_{N}\right)$ yields an ascending chain

$$
0=\mathcal{T}_{0} \subset \mathcal{T}_{1} \subset \mathcal{T}_{2} \subset \ldots \subset \mathcal{T}_{N} \subseteq \bmod A
$$

of torsion classes such that

$$
\mathcal{T}_{t-1}^{\perp} \cap \mathcal{T}_{t}=\operatorname{add}\left(B_{t}\right)
$$

for a unique brick $B_{t}$ with $\operatorname{End}\left(B_{t}\right)=k, \operatorname{Ext}^{1}\left(B_{t}, B_{t}\right)=0$ and such that the dimension vector of $B_{t}$ is the c-vector associated with the vertex $i_{t}, 1 \leqslant t \leqslant N$. Moreover, the green sequence $\mathbf{i}$ is maximal if and only if $\mathcal{T}_{N}=\bmod A$. 
Notice that the theorem admits an obvious generalization to arbitrary (red and green) sequences of mutations (cf. section 7.6 of [78]). We refer to [53] for applications of the theorem to the study of lattice properties of oriented exchange graphs.

Corollary 5.2 (Brüstle-Dupont-Pérotin [11]). If $Q$ admits a reddening (in particular a maximal green) sequence, then $A$ is finite-dimensional.

Proof. By the theorem, each object of $\bmod A$ admits a finite zig-zag-filtration, whose subquotients are finite direct sums of the finitely many modules $B_{t}, 1 \leqslant t \leqslant N$. So in this case, there is a uniform bound on the Loewy length of the finite-dimensional $A$-modules, which implies that $A$ itself is finite-dimensional $[49,85]$, a fact proved in a different manner in [11].

Recall that the Hasse quiver Hasse $(P)$ of a poset $P$ has as vertices the elements of $P$ and an arrow $x \rightarrow y$ for each minimal inequality $x<y$, i.e. we have $x<y$ and whenever $x \leqslant z \leqslant y$, we have $x=z$ or $z=y$. It is immediate from the equality (5.1.1) that the inclusions $\mathcal{T}_{t-1} \subset \mathcal{T}_{t}$ in the chain of torsion classes associated to a green sequence are minimal inclusions. Thus, the chain yields a path starting at 0 in the Hasse quiver Hasse(tors $A$ ) of the poset of torsion classes tors $A$ of $\bmod A$. The following theorem is an immediate consequence of the results of Adachi-Iyama-Reiten [1] and Demonet-Iyama-Jasso [32].

TheOREm 5.3. Suppose $\operatorname{dim} A<\infty$. Then Nagao's map taking a green sequence $\mathbf{i}$ to the chain of torsion classes $\left(\mathcal{T}_{t}\right)$ is a bijection from the set of green sequences for $Q$ to the set of paths starting at 0 in the Hasse quiver of torsion classes of $\bmod A$.

Proof. Recall that a torsion class $\mathcal{T}$ is functorially finite if for each module $M$, there is a morphism $M \rightarrow T$ with $T \in \mathcal{T}$ such that each morphism $M \rightarrow T^{\prime}$ with $T^{\prime} \in \mathcal{T}$ factors through $M \rightarrow T$. Let us write ftors $A$ for the poset of functorially finite torsion classes in $\bmod A$. It follows from Theorem 4.1 of [1], cf. also Theorem 4.9 of [16], that Nagao's map is a bijection from the set of green sequences onto the set of paths starting at 0 in the quiver Hasse(ftors $A$ ). It follows from Theorem 1.3 of [32] that immediate successors and predecessors in Hasse(tors $A$ ) of functorially finite torsion classes are functorially finite. Thus the inclusion Hasse(ftors $A) \subset$ Hasse(tors $A$ ) induces an isomorphism of the connected component of 0 in Hasse(ftors $A$ ) onto the connected component of 0 in Hasse(tors $A$ ). The claim follows.

Though it is not necessary for the proof of Muller's theorem, it is an interesting question to ask which sequences of bricks $\left(B_{t}\right)$ are associated with maximal green sequences of $Q$. The following remarkably simple criterion is proved for maximal green sequences of cluster-finite quivers by Igusa in Corollary 2.14 of [65]. A more general statement concerning (possibly infinite) chains in the poset of torsion classes of a finite-dimensional algebra is proved by Demonet in Appendix A. Applications to the construction of maximal green sequences for representation-finite cluster-tilted algebras are given in the appendix to [65] for type $A$ and in [99] for arbitrary type.

Theorem 5.4 (Igusa [65], Demonet App. A). Suppose $\operatorname{dim} A<\infty$. A sequence of bricks $B_{1}, \ldots, B_{N}$ is associated with a maximal green sequence for $Q$ if and only if

$$
\operatorname{Hom}\left(B_{i}, B_{j}\right)=0 \text { for all } i<j
$$

and the sequence cannot be refined keeping this condition.

To conclude the proof of Muller's theorem, we need one more recent result on torsion classes. By definition, if $R$ and $R^{\prime}$ are quivers, a contraction $R \rightarrow R^{\prime}$ is a functor from the path category of $R$ to that of $R^{\prime}$ which maps each arrow to an arrow or an identity morphism.

Theorem 5.5 (Demonet-Iyama-Reading-Reiten-Thomas [33]). Let A be a finite-dimensional algebra and $I \triangleleft A$ a 2 -sided ideal. Then the map $\mathcal{T} \mapsto \mathcal{T} \cap \bmod (A / I)$ induces a contraction

$$
\text { Hasse (tors } A) \rightarrow \text { Hasse (tors } A / I) \text {. }
$$

Proof. Let us first recall from Theorem 3.3 of [33], cf. also Theorem 1.0.2 of [6], that an inclusion of torsion classes $\mathcal{S} \subseteq \mathcal{T}$ is minimal if and only if there is a unique (up to isomorphism) brick in $\mathcal{S}^{\perp} \cap \mathcal{T}$ and that it is an equality if and only if there is no brick in $\mathcal{S}^{\perp} \cap \mathcal{T}$. Now let $\mathcal{S} \subset \mathcal{T}$ be 
a minimal inclusion of torsion classes in $\bmod A$. Let $\mathcal{S}_{I}=\mathcal{S} \cap \bmod (A / I)$ and $\mathcal{T}_{I}=\mathcal{T} \cap \bmod (A / I)$. Let $M$ be a module in $\mathcal{S}_{I}^{\perp} \cap \mathcal{T}_{I}$. Let $M_{\mathcal{S}} \subseteq M$ be its maximal submodule in $\mathcal{S}$. Then $M_{\mathcal{S}}$ clearly belongs to $\mathcal{S}_{I}$. Since $M$ is right orthogonal to $\mathcal{S}_{I}$, we have $M_{\mathcal{S}}=0$. Thus, the module $M$ belongs to $\mathcal{S}^{\perp} \cap \mathcal{T}$ and we have $\mathcal{S}_{I}^{\perp} \cap \mathcal{T}_{I} \subset \mathcal{S}^{\perp} \cap \mathcal{T}$. Thus, the subcategory $\mathcal{S}_{I}^{\perp} \cap \mathcal{T}_{I}$ contains either zero or one brick and the inclusion $\mathcal{S}_{I} \subseteq \mathcal{S}_{I}$ is either an equality or minimal.

REMARK 5.6. As explained in section 3.2 of [33], a brick $B$ is associated with each minimal inclusion of torsion classes $\mathcal{S} \subset \mathcal{T}$ of $\bmod A$ and the proof shows that such an inclusion is mapped to a minimal inclusion in $\bmod (A / I)$ if and only if $B$ belongs to $\bmod (A / I)$ (i.e. $B$ is annihilated by I).

We can now conclude: Suppose that $Q$ has a maximal green sequence and that $Q^{\prime} \subseteq Q$ is a full subquiver. Let $e$ be the sum of the lazy idempotents $e_{i}$ associated with the vertices not in $Q_{0}^{\prime}$. Clearly, the algebra $\widehat{k Q^{\prime}}$ is isomorphic to the quotient of $\widehat{k Q}$ by the two-sided ideal generated by $e$. Let $W^{\prime} \in \widehat{k Q^{\prime}}$ be the image of $W$ under the projection $\widehat{k Q} \rightarrow \widehat{k Q^{\prime}}$. By Corollary 22 of [86] (the published version!), the potential $W^{\prime}$ is non degenerate on $Q^{\prime}$. Let $A^{\prime}$ be the Jacobi algebra of $\left(Q^{\prime}, W^{\prime}\right)$. Clearly, the algebra $A^{\prime}$ is isomorphic to the quotient of $A$ by the two-sided ideal generated by $e$. By the theorem above, the map

$$
\text { Hasse }(\operatorname{tors} A) \rightarrow \text { Hasse }\left(\operatorname{tors} A^{\prime}\right), \mathcal{T} \mapsto \mathcal{T} \cap \bmod A^{\prime}
$$

is a contraction and clearly, it takes 0 to 0 and $\bmod A$ to $\bmod A^{\prime}$. Thus, it maps a finite path from 0 to $\bmod A$ to a finite path from 0 to $\bmod A^{\prime}$. By Theorem 5.3, we obtain a maximal green sequence for $Q^{\prime}$.

REMARK 5.7. By comparing remarks 4.3 and 5.6 we see that both proofs yield the same explicit recipe for constructing the induced maximal green sequence.

\section{Comparing the proofs}

Let us emphasize that the statement of Muller's theorem 4.2 and the proof via scattering diagrams given by him in [93] go through for valued quivers. In contrast, our representationtheoretic proof only works for the classes of valued quivers treated by Demonet [30, 31].

When trying to compare the two proofs we are naturally lead to the problem of relating torsion classes to scattering diagrams. A first step towards its solution was taken by Bridgeland [10] who associates a scattering diagram (with values in a motivic Hall algebra) to each finite quiver with (polynomial) relations and without loops or 2-cycles. By a result of Hua-Song [63], each potential on a quiver $Q$ whose (complete) Jacobi-algebra is finite-dimensional is right-equivalent to a polynomial potential $W$. So if the morphism from the non complete Jacobi algebra of $(Q, W)$ to the complete Jacobi algebra is bijective (which is not automatic even if the non complete Jacobi algebra is finite-dimensional!), then Bridgeland's construction applies to this case. In particular, it often applies when the quiver admits a reddening sequence. The problem of comparing Bridgeland's stability scattering diagram with the cluster scattering diagram of Gross-Hacking-Keel-Kontsevich $[60]$ is the subject of ongoing research $[\mathbf{2 8}, \mathbf{1 0 3}]$. Other topics closely related to the representationtheoretic proof are the investigation of chains of torsion classes induced by stability conditions $[65,64,14,4,13,111]$, the wall and chamber structure of the space of stability conditions [15] and the study of semi-invariants and picture groups $[67,66,68]$.

\section{Appendix A. Maximal chains of torsion classes, by Laurent Demonet}

Let $A$ be a finite-dimensional algebra over a field $k$. We consider the category $\bmod A$ of finitedimensional right $A$-modules and its lattice tors $(A)=\operatorname{tors}(\bmod A)$ of torsion classes $(\operatorname{cf}$. section 5$)$. It is a complete lattice, i.e. the associated category has all limits, called meets, and all colimits, called joins. In particular, for each set of modules $X \subseteq \bmod A$, there is a smallest torsion class $\mathrm{T}(X)$ containing $X$. An element $x$ of a complete poset is completely join irreducible if it is not the join of an arbitrary family of elements $<x$. For a poset $P$, we denote by Hasse $(P)$ the Hasse quiver of $P$, whose vertices are the elements of $P$ and which has an arrow $x \rightarrow y$ if $x<y$ and $x=z$ or $z=y$ 
whenever $x \leqslant z \leqslant y$ (it is opposite to the Hasse quiver of [33]). If $P$ is complete, we denote by $\operatorname{cjirr}(P)$ the subposet of completely join irreducible elements.

A brick is an $A$-module whose endomorphisms form a division algebra. We write $\operatorname{brick}(A)$ for the set of isomorphism classes of bricks of $\bmod A$.

A chain of torsion classes is a totally ordered subposet of tors $(A)$. Chains of torsion classes are ordered by inclusion. Using the results of [33] we will describe the (possibly infinite!) maximal chains of torsion classes in terms of the bricks of $\bmod A$. Notice that part of the results of [33] were independently obtained in [6].

Let $I$ be a totally ordered set. An $I$-chain of bricks is a map

$$
S_{\bullet}: I \rightarrow \operatorname{brick}(A), i \mapsto S_{i}
$$

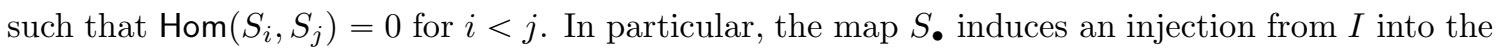
set of isomorphism classes of bricks. If $I^{1}$ and $I^{2}$ are totally ordered sets and $S_{\bullet}^{1}, S_{\bullet}^{2}$ are chains of bricks indexed by $I^{1}$ and $I^{2}$, we write $S_{\bullet}^{1} \leqslant S_{\bullet}^{2}$ if there is an increasing inclusion $\iota: I^{1} \rightarrow I^{2}$ such that for all $i \in I^{1}$, the module $S_{i}^{1}$ is isomorphic to $S_{\iota(i)}^{2}$. Suppose we have an inequality $S_{\bullet}^{1} \leqslant S_{\bullet}^{2}$ given by $\iota$ and an inequality $S_{\bullet}^{2} \leqslant S_{\bullet}^{1}$ given by $\kappa$. Then for each $i \in I^{1}$, the module $S_{i}$ is isomorphic to $S_{\kappa(\iota(i))}$ so that $\kappa \iota(i)=i$ and $\iota$ and $\kappa$ are bijective. We define the chains of bricks $S_{\bullet}^{1}$ and $S_{\bullet}^{2}$ to be equivalent if we have $S_{\bullet}^{1} \leqslant S_{\bullet}^{2} \leqslant S_{\bullet}^{1}$.

Let $I$ be a totally ordered set and $S_{\bullet}$ an $I$-chain of bricks. We will associate with $S_{\bullet}$ a chain of torsion classes $\mathcal{T}_{\bullet}=\Phi\left(S_{\bullet}\right)$. An ideal of $I$ is a subset $j \subseteq I$ such that $l \in j$ whenever $l \leqslant i$ and $i \in j$. Let $J$ be the poset of ideals of $I$. For each $j \in J$, let $\mathcal{T}_{j}$ be the smallest torsion class containing the $S_{i}, i \in j$. It is the join of the torsion classes $\mathrm{T}\left(S_{i}\right), i \in j$. Clearly, the map $j \mapsto \mathcal{T}_{j}$ is increasing. If we have $j \subset j^{\prime}$ and $i$ belongs to $j^{\prime}$ but not to $j$, then the brick $S_{i}$ lies in $\mathcal{T}_{j^{\prime}} \cap \mathcal{T}_{j}^{\perp}$ so that the map $j \mapsto \mathcal{T}_{j}$ is strictly increasing. We define $\Phi\left(S_{\bullet}\right)$ to be the subposet of $\operatorname{tors}(A)$ formed by the $\mathcal{T}_{j}$, $j \in J$.

Proposition A.1. The map $\Phi$ induces an injective morphism from the poset of equivalence classes of chains of bricks to the poset of chains of torsion classes.

Proof. Clearly, the map $\Phi$ is a morphism of posets. Let $S_{\bullet}: I \rightarrow \operatorname{brick}(A)$ and $S_{\bullet}^{\prime}: I^{\prime} \rightarrow$ $\operatorname{brick}(A)$ be chains of bricks and $\mathcal{T}_{\bullet}=\Phi\left(S_{\bullet}\right)$ and $\mathcal{T}_{\bullet}^{\prime}=\Phi\left(S_{\bullet}^{\prime}\right)$ the associated chains of torsion classes. Let $J$ and $J^{\prime}$ be the posets of ideals of $I$ and $I^{\prime}$. We first construct an isomorphism of posets $\pi: I \stackrel{\sim}{\rightarrow} I^{\prime}$ such that for each $j \in J$, we have $\mathcal{T}_{\pi(j)}^{\prime}=\mathcal{T}_{j}$. For this, notice that $J$ and $\operatorname{tors}(A)$ are complete lattices and that the injective morphism $j \mapsto \mathcal{T}_{j}$ commutes with arbitrary joins. We can recover $I$ from the set of ideals $J$ of $I$ as the subposet of the completely join irreducible elements. Since we have isomorphisms of posets

$$
J \stackrel{\sim}{\rightarrow}\left\{\mathcal{T}_{j} \mid j \in J\right\}=\left\{\mathcal{T}_{j^{\prime}}^{\prime} \mid j^{\prime} \in J^{\prime}\right\} \stackrel{\sim}{\leftarrow} J^{\prime}
$$

we obtain an isomorphism $\pi: I \stackrel{\sim}{\rightarrow} I^{\prime}$ and it clearly satisfies $\mathcal{T}_{j}=\mathcal{T}_{\pi j}^{\prime}$ for all $j \in J$. Thus, we may assume that $S_{\bullet}$ and $S_{\bullet}^{\prime}$ are maps $I \rightarrow \operatorname{brick}(A)$ such that for each ideal $j$ of $I$, the join $\mathcal{T}_{j}$ of the classes $\mathrm{T}\left(S_{i}\right), i \in j$, coincides with $\mathcal{T}_{j}^{\prime}$. Fix an element $i$ of $I$. Let $j \subseteq I$ be the ideal of the elements $i^{\prime}<i$. Let $\mathcal{U}=\mathcal{T}_{j}=\mathcal{T}_{j}^{\prime}$. Let $\mathcal{T}$ be the torsion class $\mathcal{U} \vee \mathrm{T}\left(S_{i}\right)=\mathcal{U} \vee \mathrm{T}\left(S_{i}^{\prime}\right)$. Since $S_{i}$ belongs to $\mathcal{U}^{\perp}$, by part a) of Theorem 3.4 of [33], the torsion class $\mathcal{T}$ is completely join irreducible and the unique arrow of the Hasse quiver Hasse[ $\mathcal{U}, \bmod A]$ ending at $\mathcal{T}$ is labelled by $S_{i}$, cf. Definition 3.5 of [33]. Since we also have $\mathcal{T}=U \vee \mathrm{T}\left(S_{i}^{\prime}\right)$, the arrow is also labeled by $S_{i}^{\prime}$, which is therefore isomorphic to $S_{i}$.

Proposition A.2. Let $C$ be a maximal chain of torsion classes. Then each $\mathcal{T} \in C$ equals the join $\mathcal{V}$ of the $\mathcal{U} \in \operatorname{cjirr}(C)$ contained in $\mathcal{T}$. It also equals the join $\mathcal{V}^{\prime}$ of the classes $\mathrm{T}\left(S_{q}\right)$, where $q$ runs through the arrows of the Hasse quiver of $C \cap[0, \mathcal{T}]$.

Proof. Clearly, we have $\mathcal{V} \subseteq \mathcal{T}$. Let us show that $\mathcal{V}^{\prime}$ is contained in $\mathcal{V}$. If $q: \mathfrak{U}^{\prime} \rightarrow \mathfrak{U}$ is an arrow of the Hasse quiver of $C \cap[0, \mathcal{T}]$, it is also an arrow of Hasse $(A)$, by the maximality of $C$. Thus, the arrow has a well-defined brick label $S_{q}$. Moreover, the brick $S_{q}$ belongs to $\mathcal{U}$ and $\mathcal{U}$ is completely join irreducible. Therefore, we have $\mathrm{T}\left(S_{q}\right) \subset \mathcal{V}$ and $\mathcal{V}^{\prime} \subset \mathcal{V}$. Let us show that the inclusion $\mathcal{V}^{\prime} \subseteq \mathcal{T}$ is an equality. By Lemma 3.10 of [33], the modules in $\mathcal{T} \cap \mathcal{V}^{\prime \perp}$ are those admitting 
BERNHARD KELLER

a filtration whose subquotients are bricks in $\mathcal{T} \cap \mathcal{V}^{\prime \perp}$. Let $S$ be a brick of minimal dimension in $\mathcal{T} \cap \mathcal{V}^{\prime \perp}$. Consider the meet $\mathcal{W}$ of the torsion classes $\mathcal{W}^{\prime} \in C$ containing $\mathcal{V}^{\prime}$ and $S$. Since $C$ is a maximal chain, it is stable under meets and thus contains $\mathcal{W}$. Let $\mathcal{V}^{\prime \prime} \in C$ be a torsion class such that $\mathcal{V}^{\prime} \subseteq \mathcal{V}^{\prime \prime} \subseteq \mathcal{W}$ and $\mathcal{V}^{\prime \prime} \neq \mathcal{W}$. Let $X \in \mathcal{V}^{\prime \prime}$. Consider a morphism $f: X \rightarrow S$. The image $\operatorname{im}(f)$ is a quotient of $X$ and thus belongs to $\mathcal{T}$. It is also a submodule of $S$ and thus belongs to $\mathcal{V}^{\prime} \perp$. Thus, it belongs to $\mathcal{D} \cap \mathcal{V}^{\prime \perp}$ and has a filtration whose subquotients are bricks in $\mathcal{T} \cap \mathcal{V}^{\prime \perp}$. By the minimality of the dimension of $S$, we have $\operatorname{im}(f)=0$ or $\operatorname{im}(f)=S$. If we have $\operatorname{im}(f)=S$, then $S$ is a quotient of $X$ and belongs to $\mathcal{V}^{\prime \prime}$, which contradicts the definition of $\mathcal{W}$. Thus, we have $\operatorname{im}(f)=0$ and $\mathcal{V}^{\prime \prime} \subset{ }^{\perp} S \cap \mathcal{W}$. By the maximality of $C$, it follows that the class ${ }^{\perp} S \cap \mathcal{W}$ belongs to $C$ and there is an arrow ${ }^{\perp} S \cap \mathcal{W} \rightarrow \mathcal{S}$ in Hasse $(C)$ labeled by $S$, cf. Theorem 3.4 of [33]. So the module $S$ belongs to $\mathcal{V}$, which is a contradiction.

Theorem A.3. The map $\Phi: S_{\bullet} \mapsto \mathcal{T}$ • induces a bijection from the set of equivalence classes of maximal chains of bricks to the set of maximal chains of torsion classes.

Proof. By Proposition A.1, it only remains to prove that $\Phi$ is surjective. Let $C$ be a maximal chain of torsion classes. Let $I$ be the poset $\operatorname{cjir}(C)$. For each $i \in I$, there is a unique arrow $\mathcal{U}_{i} \rightarrow i$ in Hasse $(C)$. By the maximality, it is also an arrow of Hasse $(A)$. Let $S_{i}$ be its label, $c f$. Definition 3.5 of [33]. We claim that the map $i \mapsto S_{i}$ is an $I$-chain of bricks. Indeed, if we have $i<j$ in $I$, then $i \subseteq \mathcal{U}_{j} \subseteq j$ and so $S_{j} \in \mathcal{U}_{j}^{\perp}$ and $S_{i} \in i \subseteq \mathcal{U}_{j}$ so that we have $\operatorname{Hom}\left(S_{i}, S_{j}\right)=0$. Let $\mathcal{T}$. be the chain of torsion classes $\Phi\left(S_{\bullet}\right)$. For $\mathcal{U} \in C$, consider the ideal $j$ of $I$ formed by the $i \in I$ contained in $U$. By Proposition A.2, we have $\mathcal{U}=\mathcal{T}_{j}$. Whence an inclusion $C \subseteq \mathcal{T}_{\bullet}$. By the maximality of $C$, we actually have an equality.

\section{References}

[1] Takahide Adachi, Osamu Iyama, and Idun Reiten, $\tau$-tilting theory, Compos. Math. 150 (2014), no. 3, 415-452. 5,5

[2] Murad Alim, Sergio Cecotti, Clay Córdova, Sam Espahbodi, Ashwin Rastogi, and Cumrun Vafa, $\mathcal{N}=2$ quantum field theories and their BPS quivers., Adv. Theor. Math. Phys. 18 (2014), no. 1, 27-127 (English). $3.1,4.9$

[3] Justin Allman and Richárd Rimányi, Quantum dilogarithm identities for the square product of A-type Dynkin quivers, Math. Res. Lett. 25 (2018), no. 4, 1037-1087. 3.1

[4] P. J. Apruzzese and Kiyoshi Igusa, Stability conditions for affine type A, arXiv:1804.09100 [math.RT]. 6

[5] Aslak Bakke Buan, Osamu Iyama, Idun Reiten, and Jeanne Scott, Cluster structures for 2-Calabi-Yau categories and unipotent groups, Compos. Math. 145 (2009), no. 4, 1035-1079. 4.1

[6] Emily Barnard, Andrew T. Carroll, and Shijie Zhu, Minimal inclusions of torsion classes, arXiv:1710.08837 [math.RT]. 5, A

[7] Andre Beineke, Thomas Brüstle, and Lutz Hille, Cluster-cyclic quivers with three vertices and the Markov equation, Algebr. Represent. Theory 14 (2011), no. 1, 97-112, With an appendix by Otto Kerner. 4.8

[8] Arkady Berenstein, Sergey Fomin, and Andrei Zelevinsky, Parametrizations of canonical bases and totally positive matrices, Adv. Math. 122 (1996), no. 1, 49-149. 3.3

[9] Cluster algebras. III. Upper bounds and double Bruhat cells, Duke Math. J. 126 (2005), no. 1, 1-52. 4.1

[10] Tom Bridgeland, Scattering diagrams, Hall algebras and stability conditions, Algebr. Geom. 4 (2017), no. 5, 523-561. 6

[11] Thomas Brüstle, Grégoire Dupont, and Matthieu Pérotin, On maximal green sequences, Int. Math. Res. Not. IMRN (2014), no. 16, 4547-4586. 2.4, 4.1, 4.8, 5.2, 5

[12] Thomas Brüstle, Stephen Hermes, Kiyoshi Igusa, and Gordana Todorov, Semi-invariant pictures and two conjectures on maximal green sequences, J. Algebra 473 (2017), 80-109. 4.1, 4.6

[13] Thomas Brüstle, David Smith, and Hipolito Treffinger, Stability conditions and maximal green sequences in abelian categories, arXiv:1805.04382 [math.RT]. 6

[14] Stability conditions, $\tau$-tilting theory and maximal green sequences, arXiv:1705.08227 [math.RT]. 6

[15] — Wall and chamber structure for finite-dimensional algebras, arXiv:1805.01880 [math.RT]. 6

[16] Thomas Brüstle and Dong Yang, Ordered exchange graphs, Advances in representation theory of algebras, EMS Ser. Congr. Rep., Eur. Math. Soc., Zürich, 2013, pp. 135-193. 5

[17] Eric Bucher, Maximal green sequences for cluster algebras associated to the $n$-torus, arXiv:1412.3713 [math.CO]. 4.9

[18] Eric Bucher and John Machacek, Reddening sequences for Banff quivers and the class $\mathcal{P}$, arXiv:1807.03359 [math.AC]. 4.1 
[19] Eric Bucher, John Machacek, Evan Runburg, Abe Yeck, and Ethan Zewde, Building maximal green sequences via component preserving mutations, arXiv:1902.02262 [math.CO]. 4.1

[20] Eric Bucher, John Machacek, and Michael Shapiro, Upper cluster algebras and choice of ground ring, arXiv:1802.04835 [math.AC]. 3.4

[21] Eric Bucher and Matthew R. Mills, Maximal green sequences for cluster algebras associated with the $n$-torus with arbitrary punctures, J. Algebraic Combin. 47 (2018), no. 3, 345-356. 4.9

[22] Philippe Caldero and Frédéric Chapoton, Cluster algebras as Hall algebras of quiver representations, Comment. Math. Helv. 81 (2006), no. 3, 595-616. 3.4

[23] Peigen Cao and Fang Li, Uniformly column sign-coherence and the existence of maximal green sequences, arXiv:1712.00973 [math.RT]. 4.1

[24] Sabin Cautis and Harold Williams, Cluster theory of the coherent Satake category, arXiv:1801.08111 [math.RT]. 3.3

[25] Sergio Cecotti, Clay Córdova, and Cumrun Vafa, Braids, walls and mirrors, arXiv:1110.2115 [hep-th]. 3.1

[26] Giovanni Cerulli Irelli, Bernhard Keller, Daniel Labardini-Fragoso, and Pierre-Guy Plamondon, Linear independence of cluster monomials for skew-symmetric cluster algebras, Compos. Math. 149 (2013), no. 10, 1753-1764. 3.4

[27] Frédéric Chapoton, Sergey Fomin, and Andrei Zelevinsky, Polytopal realizations of generalized associahedra, Canad. Math. Bull. 45 (2002), no. 4, 537-566, Dedicated to Robert V. Moody. 4.1

[28] Man-Wai Cheung and Travis Mandel, Donaldson-Thomas invariants from tropical disks, arXiv:1902.05393 [math.AG]. 6

[29] E. Cormier, P. Dillery, J. Resh, K. Serhiyenko, and J. Whelan, Minimal length maximal green sequences and triangulations of polygons, J. Algebraic Combin. 44 (2016), no. 4, 905-930. 4.9

[30] Laurent Demonet, Mutations of group species with potentials and their representations. Applications to cluster algebras, arXiv:1003.5078 [math.RT]. 6

[31] Categorification of skew-symmetrizable cluster algebras, Algebras and Representation Theory 14 (2011), no. 6, 1087-1162. 6

[32] Laurent Demonet, Osamu Iyama, and Gustavo Jasso, $\tau$-tilting finite algebras, bricks, and g-vectors, Int. Math. Res. Not. IMRN (2019), no. 3, 852-892. 5, 5

[33] Laurent Demonet, Osamu Iyama, Nathan Reading, Idun Reiten, and Hugh Thomas, Lattice theory of torsion classes, arXiv:1711.01785 [math.RT]. 1, 1, 5.5, 5, 5.6, A, A, A, A

[34] Harm Derksen and Theodore Owen, New graphs of finite mutation type, Electron. J. Combin. 15 (2008), no. 1, Research Paper 139, 15. 4.9

[35] Harm Derksen, Jerzy Weyman, and Andrei Zelevinsky, Quivers with potentials and their representations I: Mutations, Selecta Mathematica 14 (2008), 59-119. 2.2, 5

[36] —, Quivers with potentials and their representations II: Applications to cluster algebras, J. Amer. Math. Soc. 23 (2010), 749-790. 2.3, 2.2

[37] Spencer E. Dickson, A torsion theory for Abelian categories, Trans. Amer. Math. Soc. 121 (1966), 223-235. 5

[38] G. Dupont, Generic variables in acyclic cluster algebras, J. Pure Appl. Algebra 215 (2011), no. 4, 628-641. 3.4

[39] L. Faddeev and A. Yu. Volkov, Abelian current algebra and the Virasoro algebra on the lattice, Phys. Lett. B 315 (1993), no. 3-4, 311-318. 3.1

[40] L. D. Faddeev and R. M. Kashaev, Quantum dilogarithm, Modern Phys. Lett. A 9 (1994), no. 5, 427-434. 3.1

[41] Jiarui Fei, Tensor product multiplicities via upper cluster algebras, arXiv:1603.02521 [math.RT]. 1, 3.4

[42] Anna Felikson, Michael Shapiro, and Pavel Tumarkin, Cluster algebras of finite mutation type via unfoldings., Int. Math. Res. Not. 2012 (2012), no. 8, 1768-1804 (English). 2.1

[43] - Skew-symmetric cluster algebras of finite mutation type., J. Eur. Math. Soc. (JEMS) 14 (2012), no. 4, 1135-1180 (English). 2.1

[44] Vladimir V. Fock and Alexander B. Goncharov, Cluster ensembles, quantization and the dilogarithm, Annales scientifiques de l'ENS 42 (2009), no. 6, 865-930. 3.5

[45] Sergey Fomin, Michael Shapiro, and Dylan Thurston, Cluster algebras and triangulated surfaces. I. Cluster complexes, Acta Math. 201 (2008), no. 1, 83-146. 2.1, 3.1, 4.9

[46] Sergey Fomin and Andrei Zelevinsky, Cluster algebras. I. Foundations, J. Amer. Math. Soc. 15 (2002), no. 2, 497-529 (electronic). 1, 2.1

[47] _ Cluster algebras. II. Finite type classification, Invent. Math. 154 (2003), no. 1, 63-121. 4.1

[48] _ Cluster algebras IV: Coefficients, Compositio Mathematica 143 (2007), 112-164. 2.1

[49] P. Gabriel, Représentations indécomposables, Séminaire Bourbaki, 26e année, no. 444, 1973/74. 5

[50] Peter Gabriel, Indecomposable representations. II, Symposia Mathematica, Vol. XI (Convegno di Algebra Commutativa, INDAM, Rome, 1971), Academic Press, London, 1973, pp. 81-104. 5

[51] Davide Gaiotto, Gregory W. Moore, and Andrew Neitzke, Wall-crossing, Hitchin systems, and the WKB approximation, arXiv:0907.3987 [physics.hep-th]. 1, 3.1

[52] —, Wall-crossing, Hitchin systems, and the WKB approximation, Adv. Math. 234 (2013), 239-403. 1

[53] Alexander Garver and Thomas McConville, Lattice properties of oriented exchange graphs and torsion classes, Algebr. Represent. Theory 22 (2019), no. 1, 43-78. 5 
[54] Alexander Garver, Thomas McConville, and Khrystyna Serhiyenko, Minimal length maximal green sequences, Sém. Lothar. Combin. 78B (2017), Art. 16, 12. 4.9

[55] _ Minimal length maximal green sequences, Adv. in Appl. Math. 96 (2018), 76-138. 4.9

[56] Alexander Garver and Gregg Musiker, On maximal green sequences for type $\mathbb{A}$ quivers, J. Algebraic Combin. 45 (2017), no. 2, 553-599. 2.2, 4.1, 4.9

[57] Christof Geiß, Bernard Leclerc, and Jan Schröer, Kac-Moody groups and cluster algebras, Adv. Math. 228 (2011), no. 1, 329-433. 4.1

[58] _ Generic bases for cluster algebras and the Chamber ansatz, J. Amer. Math. Soc. 25 (2012), no. 1, 21-76. 1, 3.3, 3.4

[59] Alexander Goncharov and Linhui Shen, Donaldson-Thomas transformations of moduli spaces of G-local systems, Adv. Math. 327 (2018), 225-348. MR 37619953.1

[60] Mark Gross, Paul Hacking, Sean Keel, and Maxim Kontsevich, Canonical bases for cluster algebras, J. Amer. Math. Soc. 31 (2018), no. 2, 497-608. 1, 2.2, 3.5, 4.1, 6

[61] Mark Gross and Bernd Siebert, From real affine geometry to complex geometry, Ann. of Math. (2) 174 (2011), no. $3,1301-1428.4 .1$

[62] Stephen Hermes and Kiyoshi Igusa, The no gap conjecture for tame hereditary algebras, J. Pure Appl. Algebra 223 (2019), no. 3, 1040-1053. 3.1

[63] Zheng Hua and Gui-Song Zhou, Noncommutative Mather-Yau theorem and its applications to Calabi-Yau algebras and birational geometry, arXiv:1803.06128 [math.AG]. 6

[64] Kiyoshi Igusa, Linearity of stability conditions, arXiv:1706.06986 [math.RT]. 6

[65] _ Maximal green sequences for cluster-tilted algebras of finite representation type, arXiv:1706.06503 [math.RT]. 1, 5, 5.4, 6

[66] Kiyoshi Igusa, Kent Orr, Gordana Todorov, and Jerzy Weyman, Modulated semi-invariants, arXiv:1507.03051 [math.RT]. 6

[67] Kiyoshi Igusa, Kent Orr, Gordana Todorov, and Jerzy Weyman, Cluster complexes via semi-invariants., Compos. Math. 145 (2009), no. 4, 1001-1034 (English). 6

[68] Kiyoshi Igusa, Gordana Todorov, and Jerzy Weyman, Picture groups of finite type and cohomology in type $A_{n}$, arXiv:1609.02636 [math.RT]. 6

[69] Kiyoshi Igusa and Ying Zhou, The formula for the permutation of mutation sequences in $A_{n}$ straight orientation, arXiv:1606.00958 [math.RT]. 2.2

[70] _ Maximal green sequences of quivers with multiple edges, arXiv:1902.07375 [math.RT]. 4.1

[71] Colin Ingalls and Hugh Thomas, Noncrossing partitions and representations of quivers, Compos. Math. 145 (2009), no. 6, 1533-1562. 1, 5

[72] Ryoichi Kase, Remarks on lengths of maximal green sequences for quivers of type $\widetilde{A}_{n, 1}$, arXiv:1507.02852 [math.RT]. 4.9

[73] Bernhard Keller, Cluster algebras and derived categories, arXiv:1202.4161 [math.RT]. 3.1

[74] — Quantum dilogarithm identities from quiver mutations, video of a talk given at the meeting 'Test problems for the theory of finite dimensional algebras', Banff, September 9, 2010,. 1, 2.2, 3.1

[75] _ Quiver mutation and combinatorial DT-invariants, corrected version of a submission to FPSAC 2013, arXiv:1709.03143 [math.CO]. 2.2, 3.1, 4.1

[76] — Quiver mutation in Javascript and Java, Java applet available at https://webusers.imj-prg.fr/ bernhard.keller/quivermutation/. 2.1

[77] EMS Ser. Congr. Rep., Eur. Math. Soc., Zürich, 2011, pp. 85-116. MR 2931896 1, 2.2, 3.1, 3.3

[78] _ Cluster algebras and derived categories, Derived categories in algebraic geometry, EMS Ser. Congr. Rep., Eur. Math. Soc., Zürich, 2012, pp. 123-183. MR 3050703 2.1, 3.3, 5, 5

[79] _ The periodicity conjecture for pairs of Dynkin diagrams, Ann. of Math. (2) 177 (2013), no. 1, 111-170. 3.1

[80] Hyun Kyu Kim and Masahito Yamazaki, Comments on Exchange Graphs in Cluster Algebras, Exp. Math. 29 (2020), no. 1, 79-100. 3.1

[81] Maxim Kontsevich and Yan Soibelman, Stability structures, Donaldson-Thomas invariants and cluster transformations, arXiv:0811.2435 [math.AG]. 1, 3.1

[82] _ Affine structures and non-Archimedean analytic spaces, The unity of mathematics, Progr. Math., vol. 244, Birkhäuser Boston, Boston, MA, 2006, pp. 321-385. 4.1

[83] _ Motivic Donaldson-Thomas invariants: summary of results, Mirror symmetry and tropical geometry, Contemp. Math., vol. 527, Amer. Math. Soc., Providence, RI, 2010, pp. 55-89. 3.1

[84] Cohomological Hall algebra, exponential Hodge structures and motivic Donaldson-Thomas invariants, Commun. Number Theory Phys. 5 (2011), no. 2, 231-352. 3.1

[85] Henning Krause and Dieter Vossieck, Length categories of infinite height, arXiv:1702.05415 [math.RT]. 5, 5

[86] Daniel Labardini-Fragoso, Quivers with potentials associated to triangulated surfaces, Proc. Lond. Math. Soc. (3) 98 (2009), no. 3, 797-839. 1, 4.9, 5

[87] Sefi Ladkani, On cluster algebras from once punctured closed surfaces, arXiv:1310.4454 [math.RT]. 4.9

[88] John W. Lawson and Matthew R. Mills, Properties of minimal mutation-infinite quivers, J. Combin. Theory Ser. A 155 (2018), 122-156. 4.9 
[89] R. J. Marsh and J. S. Scott, Twists of Plücker coordinates as dimer partition functions, Comm. Math. Phys. 341 (2016), no. 3, 821-884. 3.3

[90] Robert Marsh, Markus Reineke, and Andrei Zelevinsky, Generalized associahedra via quiver representations, Trans. Amer. Math. Soc. 355 (2003), no. 10, 4171-4186 (electronic). 1, 5

[91] Matthew R. Mills, On the relationship between green-to-red sequences, local-acyclicity, and upper cluster algebras, arXiv:1804.00479 [math.RT]. 3.4

[92] Matthew R. Mills, Maximal green sequences for quivers of finite mutation type, Adv. Math. 319 (2017), 182-210. 4.10

[93] Greg Muller, The existence of a maximal green sequence is not invariant under quiver mutation, Electron. J. Combin. 23 (2016), no. 2, Paper 2.47, 23. 2.2, 4.2, 4.7, 4.8, 6

[94] Greg Muller and David E. Speyer, The twist for positroid varieties, Proc. Lond. Math. Soc. (3) 115 (2017), no. 5, 1014-1071. 3.3

[95] Gregg Musiker and Christian Stump, A compendium on the cluster algebra and quiver package in sage, arXiv:1102.4844 [math.CO]. 2.1

[96] Kentaro Nagao, Quantum dilogarithm identities, RIMS Kokyuroku Bessatsu B28 (2011), 164-170. 3.1

[97] _ Donaldson-Thomas theory and cluster algebras, Duke Math. J. 162 (2013), no. 7, 1313-1367. 2.2, 5

[98] Tomoki Nakanishi and Andrei Zelevinsky, On tropical dualities in cluster algebras, Algebraic groups and quantum groups, Contemp. Math., vol. 565, Amer. Math. Soc., Providence, RI, 2012, pp. 217-226. 2.2

[99] Alireza Nasr-Isfahani, Maximal forward hom-orthogonal sequences for cluster-tilted algebras of finite type, arXiv:1804.05361 [math.RT]. 5

[100] Pierre-Guy Plamondon, Cluster characters, arXiv:1610.07546 [math.RT]. 3.4

[101] _ Cluster algebras via cluster categories with infinite-dimensional morphism spaces, Compositio Mathematica 147 (2011), 1921-1954. 2.2

[102] _ Generic bases for cluster algebras from the cluster category, Int. Math. Res. Not. IMRN (2013), no. 10, 2368-2420. 3.4

[103] Fan Qin, Bases for upper cluster algebras and tropical points, arXiv:1902.09507 [math.RT]. 1, 3.6, 6

[104] Yu Qiu, C-sortable words as green mutation sequences, Proc. Lond. Math. Soc. (3) 111 (2015), no. 5, 10521070. 5

[105] Markus Reineke, Poisson automorphisms and quiver moduli, J. Inst. Math. Jussieu 9 (2010), no. 3, 653-667. 3.1

[106] Konstanze Rietsch and Lauren Williams, Newton-Okounkov bodies, cluster duality, and mirror symmetry for Grassmannians, arXiv:1712.00447 [math.AG]. 3.3

[107] Ahmet I. Seven, Maximal green sequences of exceptional finite mutation type quivers, SIGMA Symmetry Integrability Geom. Methods Appl. 10 (2014), Paper 089, 5. 4.9

[108] _ Maximal green sequences of skew-symmetrizable $3 \times 3$ matrices, Linear Algebra Appl. 440 (2014), 125-130. 4.8

[109] Linhui Shen and Daping Weng, Cluster structures on double Bott-Samelson cells, arXiv:1904.07992 [math.AG]. 3.1

[110] Hugh Thomas, The Tamari lattice as it arises in quiver representations, Associahedra, Tamari lattices and related structures, Prog. Math. Phys., vol. 299, Birkhäuser/Springer, Basel, 2012, pp. 281-291. 1

[111] Hipolito Treffinger, An algebraic approach to Harder-Narasimhan filtrations, arXiv:1810.06322 [math.CT]. 6

[112] Alexander Yu. Volkov, Pentagon identity revisited, Int. Math. Res. Not. IMRN (2012), no. 20, 4619-4624. MR 29896153.1

[113] Daping Weng, Donaldson-Thomas transformation of double Bruhat cells in general linear groups, arXiv:1606.01948 [math.AG]. 3.1

[114] _ Donaldson-Thomas transformation of double Bruhat cells in semisimple Lie groups, arXiv:1611.04186 [math.AG], to appear in Ann. Scient. ENS. 3.1

[115] - Donaldson-Thomas transformation of Grassmannian, arXiv:1603.00972 [math.RT]. 3.1

[116] Dan Xie, BPS spectrum, wall crossing and quantum dilogarithm identity, Adv. Theor. Math. Phys. 20 (2016), no. 3, 405-524. 3.1

[117] Don Zagier, Polylogarithms, Dedekind zeta functions and the algebraic K-theory of fields, Arithmetic algebraic geometry (Texel, 1989), Progr. Math., vol. 89, Birkhäuser Boston, Boston, MA, 1991, pp. 391-430. 3.1

B. Keller: Université Paris Diderot - Paris 7, Sorbonne Université, UfR de Mathématiques, CNRS, Institut de Mathématiques de Jussieu-Paris Rive Gauche, imJ-PRG, Bâtiment Sophie Germain, 75205 Paris Cedex 13, France

Email address: bernhard.keller@imj-prg.fr

$U R L:$ https://webusers.imj-prg.fr/〜 bernhard.keller/

L. Demonet: Graduate School of Mathematics, Nagoya University, Chikusa-ku, Nagoya, 464-8602 JAPAN

Email address: Laurent.Demonet@normalesup.org

URL: http://www.math.nagoya-u.ac.jp/ demonet/ 